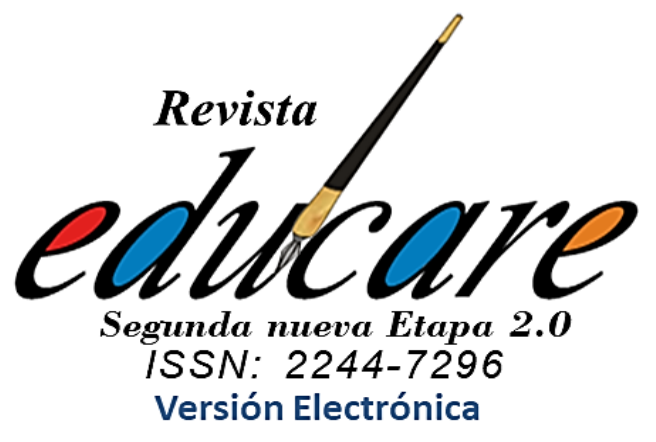

Volumen 24 № 3 Septiembre-Diciembre 2020

(488-502)

Omaira Esther Bolaño Muñoz

ORCID: https://orcid.org/0000-0001-5666-8542

\section{SECRETARÍA DE EDUCACIÓN \\ DEPARTAMENTAL DEL MAGDALENA \\ COLOMBIA}

Estudiante de Doctorado en Educación, Universidad Pedagógica Experimental Libertador de Venezuela; Magister en Educación, Universidad Autónoma del Caribe de Colombia; Especialista en Edumática, Universidad Autónoma de Colombia ;Licenciada en Matemáticas y Física Universidad del Magdalena, Colombia; Docente adscrita a la Secretaría de Educación Departamental del Magdalena de Colombia. Email: omairabola1801@hotmail.com
REPÚBLICA BOLIVARIANA DE VENEZUELA UNTVERSIDAD PEDAGÓGICA EXPERIMENTAL LIBERTADOR INSTITUTO PEDAGÓGICO DE BARQUISIMETO "LUIS BELTRÁN PRIETO FIGUEROA" SUBDIRECCIÓN DE INVESTIGACIÓN Y POSGRADO BARQUISIMETOESTADO LARA

\title{
EL CONSTRUCTIVISMO: MODELO PEDAGÓGICO PARA LA ENSEÑANZA DE LAS MATEMÁTICAS
}

Recibido:

20-05-2019

Aceptado:

04-08-2020 

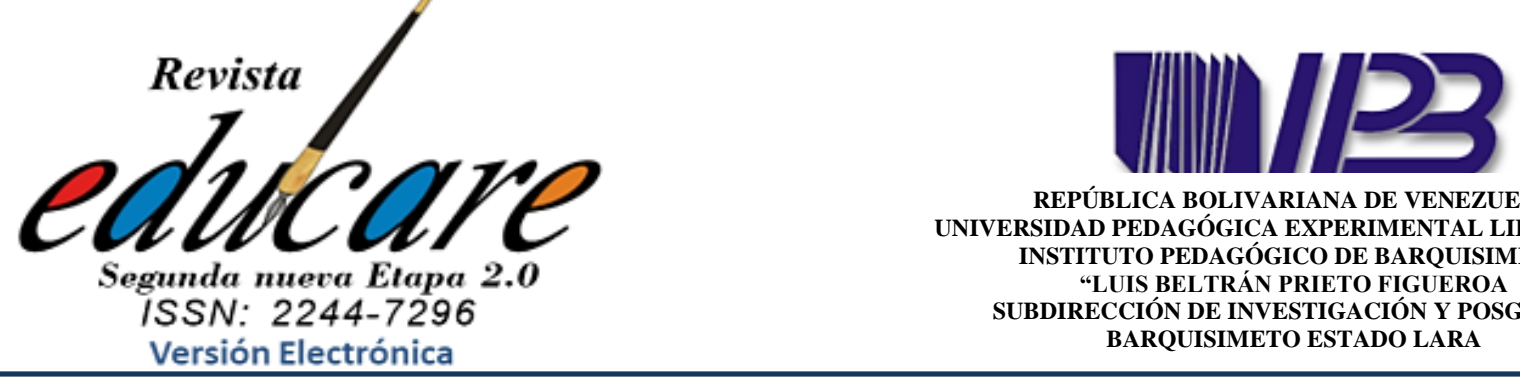

REPÚBLICA BOLIVARIANA DE VENEZUELA UNIVERSIDAD PEDAGÓGICA EXPERIMENTAL LIBERTADOR INSTITUTO PEDAGÓGICO DE BARQUISIMETO "LUIS BELTRÁN PRIETO FIGUEROA

\section{EL \\ CONSTRUCTIVISMO: MODELO PEDAGOGICO PARA LA ENSENANZA DE LAS MATEMÁTICAS}

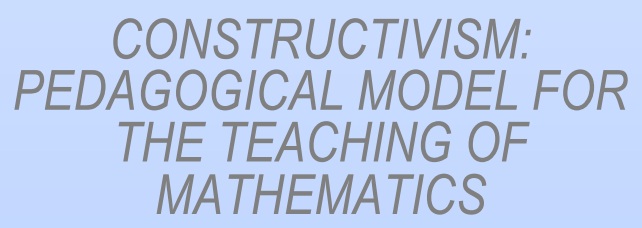

\section{Resumen}

En los albores de la modernidad la educación es considerada un factor determinante en el desarrollo social y científico, entendiéndose que los saberes se construyen desde un contexto escolarizado - contextualizado, donde convergen estudiantes, docentes, instituciones, familia y sociedad. En el presente ensayo, se hace un análisis reflexivo sobre las teorías constructivistas como modelo pedagógico para la enseñanza de las matemáticas. Se utilizó el método hermenéutico, como modo de comprensión que no está en el ser individual, sino en el ser histórico, de lo que se traduce que la hermenéutica no se ceñirá en entender al otro sino en el entenderse con el otro en un contexto determinado. Se fundamenta en la interpretación de: discurso, información y realidad presentada. Las consideraciones finales del ensayo evidenciaron que los postulados constructivistas forman parte esencial de la transformación educativa para la enseñanza de las matemáticas, partiendo de la idea de que el modelo constructivista se enriquece de las diversas aportaciones de la diversidad de áreas del saber; como son la filosofía, la psicología y la pedagogía.

Palabras clave: constructivismo; modelo pedagógico; matemáticas; enseñanza.

\begin{abstract}
At the dawn of modernity, education is considered a determining factor in social and scientific development, understanding that knowledge is built from a schooled - contextualized context, where students, teachers, institutions, family and society converge. In this essay, a reflective analysis is made of constructivist theories as a pedagogical model for the teaching of mathematics. The hermeneutical method was used, as a way of understanding that is not in the individual being, but in the historical being, which means that hermeneutics will not limit itself to understanding the other but to understanding the other in a given context . It is based on the interpretation of: speech, information and reality presented. The final considerations of the essay showed that the constructivist postulates are an essential part of the educational transformation for the teaching of mathematics, based on the idea that the constructivist model is enriched by the diverse contributions of the diversity of areas of knowledge; such as philosophy, psychology and pedagogy.
\end{abstract}

Keywords: constructivism; pedagogical model; mathematics; teaching. 


\section{INTRODUCCIÓN}

Hoy en día las sociedades están sometidas a trasformaciones tecnológicas y científicas que a su vez inducen un cambio de pensamiento en las sociedades y los individuos. Por ello todas las disciplinas científicas deben acoger estas nuevas concepciones de progresos y ajustar sus procesos para incorporarse al desarrollo de cada nación. En este sentido, la educación es entendida como el ámbito del quehacer humano más idóneo para gestar ajustes en los mercados y sociedades, ya que funge como mecanismo de formación del nuevo ciudadano. No obstante, los sistemas educativos enmarcan sus programas de estudios a la adaptación y adopción de un nuevo paradigma que vincule los procesos académicos a la realidad social.

Sin embargo, no en todas las cátedras se ven los resultados de las reformas educativas, y en el caso de las matemáticas siguen siendo vista como un proceso complejo, aburrido y poco funcional, donde sólo importa aprender las operaciones básicas (suma, resta, multiplicación y división), en este sentido, Payares (2016) afirma que no ha existido, a través de la historia de la educación asignatura que genere tantos dolores de cabeza al momento de ofrecer mecanismos de apropiación del conocimiento como los que genera la enseñanza de las matemáticas. Mientras que Albornos (2017, p. 22), afirma "en Colombia la educación apunta a la calidad por lo tanto hay que reinventar las formas de abordar los procesos educativos". De allí metodologías constructivistas permiten una mayor interactividad entre los estudiantes, donde comparten experiencias y buscan razones lógicas a los fenómenos o ecuaciones, incluso se fomentan una sana competencia de saberes.

Cabe destacar que no basta con desarrollar una propuesta sobre los métodos y las técnicas de aprendizaje, sino que resulta imperativo usarlas de forma apropiada, y para ello es necesario tener en cuenta que cada estudiante es diferente, cada uno es particularmente característico, es decir es heterogéneo. Estas afirmaciones sugieren que el estudiante en la actualidad amerita de unos métodos y estrategias de aprendizajes novedosas, interactivos que contribuyan a desarrollar habilidades y competencias cognitivas y no seguir estancados en los mismos métodos de hace diez o veinte años. Las nuevas generaciones requieren de metodologías educativas que se adhieran a sus necesidades reales, contribuyan a su desarrollo pleno y al desarrollo del pensamiento lógico matemático para que puedan hacer frente a las situaciones que debe afrontar en su cotidianidad.

Dentro de este contexto, los problemas a los cuales se enfrentan los docentes de las instituciones educativas del Municipio Zona Bananera del Departamento del Magdalena de 
Colombia, en su praxis educativa, no es a la selección de las propuestas pedagógicas sino a las formas de aplicación de las mismas, ya que la efectividad de los resultados esperados depende de los métodos y estrategias de enseñanza que se adopten, es decir, que se geste un aprendizaje significativo, en este caso en las matemáticas.

De esta forma, los docentes de estas instituciones tienen un gran reto por delante, ajustar los procesos educativos a un modelo pedagógico con predominio en el constructivismo para que la educación sea funcional y práctica, aunado a esto promover una educación que se ajuste a la realidad social.

En ese sentido se pretende analizar el constructivismo como modelo pedagógico para la enseñanza de las matemáticas, para ello se realizó una revisión documental, de esta forma la investigación no se enfocó en medir, sino cualificar, analizando el fenómeno social a partir de rasgos y teorías subyacentes determinantes, por lo que se propuso establecer el constructivismo como modelo pedagógico para la enseñanza de las matemáticas

\section{Constructivismo: Modelo para la didáctica de la matemática}

Se dice que la matemática se encuentra inmersa en cada uno de los aspectos del universo; por lo tanto, se podría concluir que cada individuo debería conocer aspectos básicos de la matemática y que este conocimiento, debería ser de fácil adquisición ya que se puede verificar o comprobar al momento de dar una mirada alrededor o analizar con lógica los mecanismos naturales del funcionamiento universal. Sin embargo, no ha existido a través de la historia de la educación, asignatura que genere tantos dolores de cabeza al momento de ofrecer mecanismos de apropiación del conocimiento como los que genera la enseñanza de las matemáticas propiamente dicha (González, 2017).

En este orden de ideas se infiere que el rechazo por el aprendizaje que se manifiesta en los estudiantes hacia esta área del saber; sin embargo, el malestar muchas veces generalizado hacia el estudio de la matemática se hace sentir en los centros educativos; en ellos, los estudiantes muestran una apatía frente a los retos que les impone la rigurosidad y abstracción característica de esta ciencia (López, 2016).

Por su parte Suárez (2012), afirma que son muchas las dificultades en el área de matemática que desde el currículo educativo que no logran afrontan la mayoría de los maestros, esas dificultades se centran en saber cómo hacer para que los estudiantes logren un aprendizaje significativo de los conceptos matemáticos, de tal forma que puedan transferir los 
conocimientos construidos en la aplicación y solución de problemas, tanto de la vida cotidiana como en contextos disciplinares específicos. Es así como en pro de una mejor calidad educativa han surgido teorías aceptadas desde la episteme de las ciencias como es el constructivismo, el cual enfatiza una enseñanza contextualizada desde las experiencias y conocimientos que el estudiante posee que pueden ser el puente para asimilar nueva información.

En este sentido la teoría de Vygotsky (1978), indica que el aprendizaje es resultado del proceso histórico y social donde el lenguaje desempeña un papel esencial. La idea del autor enfatiza la importancia que tiene el entorno social para que se produzca un aprendizaje en los estudiantes. Además, el autor sostiene que el aprendizaje es un acto activo, donde la nueva información que se recibe se incorpora a las experiencias previas y a las propias estructuras mentales.

Por su parte Piaget (1978) en su teoría explica que las personas son capaces de aprender y comprender el mundo que los rodea, describiendo cómo la inteligencia, el ambiente e incluso los genes influyen en las formas de percibir el mundo. Cabe destacar que esta teoría se fundamenta en los procesos de asimilación y acomodación; la asimilación tiene como función interiorizar la información que se recibe del entorno, profundizándola para que el cerebro la decodifique y comprenda la realidad vivida; la acomodación consiste en modificar los esquemas previos, es decir perfeccionar las ideas preconcebidas mediante la nueva información.

Desde la perspectiva de Ausubel (1973), surge lo que se conoce como aprendizaje significativo que no es más que buscar la forma de generar un conocimiento mediante la relación de información nueva con la que ya se posee, es decir con una estructura cognitiva existente. De allí que el acto educativo debe considerar la experiencia de los estudiantes como mecanismo de anclaje para la nueva información, de manera que los estudiantes puedan establecer un vínculo entre el conocimiento nuevo con sus propias experiencias, lo que puede verse como llevar el aprendizaje de los contenidos curriculares a su propia vida.

Según Dávila (2014), en la práctica, el constructivismo y los principios metodológicos que aparecen en los diseños curriculares de matemática se quedaran en plasmaciones teóricas y formulaciones de principios, siendo urgente reformar las metodologías empleadas en la enseñanza de esta área del conocimiento, donde se dé lugar a una integración con el contexto del estudiante. Al respecto Martínez (2008), señala que el constructivismo visto desde el enfoque educativo se concibe como un proceso mediante el cual el estudiante tiene mayor 
libertad y se le proporciona más autonomía, el docente lo apoya y dirige en la construcción del conocimiento; se trata de ir de lo simple (conocimiento intuitivo) a lo complejo (conocimiento formal, científico), de allí que las experiencias y el entorno social del estudiante sean crucial en el proceso educativo. Esto indica que el conocimiento surge de un saber previo.

Por ello desde los sistemas educativos se debe asumir el hecho que cuando el estudiante aprende algo nuevo, lo debe incorporar a sus experiencias previas o contexto cotidiano y a sus propias estructuras mentales para que se propicie un aprendizaje significativo. De allí que el aprendizaje viene a ser un proceso subjetivo enraizado en las experiencias del que aprende.

En virtud de esto se puede decir que las propuestas constructivistas se han convertido en el eje de una transformación fundamental de la enseñanza en el área de matemática. Por lo que cabe resaltar que el modelo constructivista no tiene una concreción didáctica uniforme debido a que se alimenta de diversas aportaciones de diferentes campos del saber; el constructivismo hunde sus raíces en postulados filosóficos, psicológicos y pedagógicos, en muchos casos divergentes. No obstante, comparte la importancia de la actividad mental constructiva del alumno (Medina Castañeda, 2011).

En este sentido se puede pensar en el constructivismo como la idea que mantiene el individuo tanto en los aspectos cognitivos y sociales del comportamiento como en los afectivos, no es un mero producto del ambiente, ni un simple resultado de sus disposiciones internas, sino una construcción propia que se va produciendo día a día como resultado de la interacción entre estos dos factores (Tünnermann, 2011).

Por otra parte, el constructivismo constituye todo un marco explicativo en el que confluyen teorías psicológicas y pedagógicas que consideran el rol del estudiante como un proceso de adquisición del conocimiento en forma dinámica, ya sean los saberes especializados como las matemáticas u otros saberes como el conocimiento de los estilos de vida, para ello es esencial que se le dé un significado a la información que recibe y luego la procese mentalmente (Vargas, 2006).

En cierto sentido esta teoría de aprendizaje se afianza en buscar el pleno desarrollo del pensamiento mediante metodologías y prácticas docentes, construir es la función que esta teoría le otorga principalmente a los procesos educativos. Es así como el constructivismo explora el aprendizaje dando una participación preponderante al estudiante, donde la experiencia de los mismos sea el marco donde se anclen los nuevos conocimientos, considerando que en el área de matemática el constructivismo proyecta un valor que no se vislumbra como algo absoluto, pues 
según esta corriente filosófica el aprendizaje es el producto de las múltiples interpretaciones que tienen los individuos de su entorno, siendo efectiva de acuerdo a las posibilidades de cada uno para interactuar y reflexionar, es allí donde el proceso educativo debe hacer énfasis, es decir, enseñar a los estudiantes a razonar la información que se les presenta para que la comprendan y puedan aplicarla tanto al contexto académico como a su cotidianidad.

Desde esta perspectiva, las matemáticas no son diferentes, ni requieren de habilidades distintivas para su aprendizaje, pero ameritan del desarrollo de competencias cognitivas específicas, como la observación, el análisis, la interpretación, entre otras. Por lo tanto, son necesarias estrategias que faciliten la adquisición de contenidos mediante actividades contextualizadas donde se pongan de manifiesto habilidades mentales para la construcción de nuevos conocimientos.

En este sentido, Melquiades (2014), expone que para el aprendizaje de las matemáticas el alumno tendrá que desarrollar su inteligencia, esta le generará mayor desarrollo de habilidades cognoscitivas propias de cada persona, para adquirir de manera fácil los contenidos que serán asimilados de una forma en la que construyan su propio aprendizaje para que sea aplicado correctamente en su vida cotidiana.

\section{Modelo pedagógico constructivista}

La nueva visión del mundo que los cambios han traído consigo requieren que los docentes adecuen sus prácticas educativas para que los procesos educativos sean cónsonos con las realidades sociales, en este sentido el rediseño de la práctica pedagógica implica que la instrucción deba basarse en el uso de casos prácticos que proporcionen experiencias de aprendizaje ricas, diversas y contextualizadas. La tarea de los docentes y formadores es diseñar ambientes de aprendizaje que ayuden a los alumnos a aprender; ya que este es un acto propio y en el que el docente solo funge como estimulados y propiciador de herramientas. Tal y como lo sostiene Piaget (2005), el mundo no es visto de la misma forma y tiene el mismo significado para todas las personas, sino que una misma situación puede tener diferentes lecturas o significados en cada individuo.

En este sentido educar no significa traspasar contenidos de un sujeto a otro, educar consiste en proporcionar las herramientas a los estudiantes para que estos construyan su propio aprendizaje. Esto guarda relación con las teorías constructivistas, ya que la experiencia y el entorno de los individuos es fundamental para su aprendizaje (Blanco, 2014). 
Dentro de este contexto del proceso educativo de las matemáticas el constructivismo se direcciona a la resolución de problemas. Suarez (2012), considera que según el constructivismo el desarrollo de los estudiantes debe estar orientado a fortalecer la zona de desarrollo próximo, es decir cada procedimiento debe estar vinculado entre sí y con la experiencia de los estudiantes, donde también se tome en cuenta el entorno donde se desarrolla socialmente.

Es así como los nuevos modelos pedagógicos se deben orientar para que durante su ejecución se obtenga información sobre el desarrollo de las competencias requeridas, de esta forma el docente podrá reestructurar las estrategias empleadas si así se requiere, considerando los resultados obtenidos. De esta manera se está en presencia de dos fases; la primera consiste en la ejecución de los planes de acción o estrategias las cuales consisten en la sucesión y combinación del proceso educativo. Lo que involucra la sucesión de la acción instruccional prevista en la planificación; la segunda fase consiste en desarrollar la habilidad para amoldar las estrategias didácticas a las condiciones de aprendizaje surgidas en el aula, en correspondencia con la valoración del saber que emerge del acto educativo y que proporciona el sustento empírico para la reflexión relativa al mejoramiento constante de la praxis educativa (Clemente, 2010).

Desde el punto de vista constructivista, el aprendizaje se trata de un proceso de desarrollo de habilidades cognitivas y afectivas, alcanzadas en ciertos niveles de maduración. Este proceso implica la asimilación y acomodación lograda por el sujeto, con respecto a la información que percibe. Se espera que esta información sea lo más significativa posible, para que pueda ser aprendida. Este proceso se realiza en interacción con los demás sujetos participantes, ya sean compañeros y docentes, para alcanzar un cambio que conduzca a una mejor adaptación al medio (Ortiz, 2015).

Siguiendo las ideas de Coloma y Tafur (1999), los modelos pedagógicos que se fundamentan en el constructivismo pedagógico parten del supuesto que la adquisición de todo conocimiento nuevo se produce a través de la movilización, por parte del sujeto de un conocimiento antiguo. El hecho de considerar que el conocimiento previo facilita el aprendizaje es un rasgo esencial del constructivismo y sustenta el aprendizaje significativo.

Igualmente, los autores antes mencionados aseguran que las metodologías basadas en el constructivismo pedagógico indican el camino para el cambio educativo, transformando éste en un proceso activo donde el alumno elabora y construye sus propios conocimientos a partir de su experiencia previa y de las interacciones que establece con el maestro y con el entorno. 
Por consiguiente, adoptar un modelo pedagógico constructivista obliga a crear ambientes de aprendizaje con características especiales, donde los estudiantes deban enfrentarse permanentemente a desempeños complejos que los comprometan con la verdadera comprensión; donde trabajen desde el principio como verdaderos científicos, comunicadores, profesionales y ciudadanos constructivos; donde los estudiantes puedan volver a utilizar aprendizajes anteriores en formas cada vez más elaboradas, conectadas y complejas; y donde puedan expresar permanentemente sus ideas, usarlas para producir acciones y productos y confrontarlas con las de los demás y con las elaboraciones que los demás hagan de ellas.

\section{El constructivismo y el docente como agente del proceso educativo}

Contextualizando esta temática, no se puede enfocar el proceso educativo solo en el estudiante dejando de lado la figura del docente, ya que este es el responsable directo del logro de los objetivos en los estudiantes, lo que quiere decir que depende de este la selección idónea, de las estrategias pedagógicas, los métodos, las herramientas para que el estudiante logre el aprendizaje que el nivel académico exige de él. Sin embargo, su posición frente a esta postura filosófica como corriente pedagógica, debe ser proporcionar al estudiante las herramientas necesarias para que este pueda alcanzar el aprendizaje.

No obstante, Salomón (2010), expresa que el docente no es ajeno al proceso de aprendizaje del estudiante por lo que está llamado a convertirse en mediador para el análisis y provocar cambios en los estudiantes. Asimismo, debe motivar y propiciar experiencias, suscitando discusiones y críticas en los alumnos. El autor viene a reforzar que, si bien es cierto que en el proceso educativo todo gira en base al estudiante, no se puede negar el hecho que el docente guía los procesos que debe asumir el discente.

Desde esta perspectiva, el docente está llamado mediar el conocimiento, en los estudiantes o las bases necesarias para acceder, lograr, alcanzar la comprensión de los contenidos y así vaya estableciendo los modelos más dinámicos para la construcción de aprendizajes más complejos. Todo esto hace imperante que el docente les asigne actividades a los estudiantes vinculándolas a contextos reales.

Carrillo (2000), opina que el maestro en la tarea de propiciador y promotor del aprendizaje, pone en juego sus concepciones epistemológicas sobre la matemática y sobre su enseñanza y aprendizaje, las cuales conforman un factor decisivo capaz de promover o no el interés de los alumnos por la asignatura y sus métodos de análisis, lo que puede tornarse en 
fortalezas u obstáculos para el desarrollo de la praxis educativa y el crecimiento profesional del maestro.

Asimismo, es necesario considerar que el enfoque constructivista le atribuye al docente el rol de mediador de los procesos cognitivos de los estudiantes. Todo indica que el constructivismo direcciona el proceso educativo, particularmente el de facilitar las herramientas que permitan al estudiante organizar la información que reciba, asimilarla como parte de su dinamismo de vida, lo que evidencia el procesamiento de la misma desde las bases cognitivas.

Dentro del constructivismo es relevante el aprendizaje significativo o contextualizado, en este caso el docente deberá conocer los conocimientos previos del dicente, es decir, se debe asegurar que el contenido a presentar pueda relacionarse con las ideas previas, ya que al conocer lo que sabe el alumno ayuda a la hora de planear qué contenidos le ayudaran a complejizar sus conocimientos previos y a adquirir nuevos.

De ahí, que partiendo de una postura constructivista y significativa el rol del docente sufre cambios. El docente deja de ser el centro de atención de la clase, cuyos objetivos están planeados desde su propia visión y en un contexto de inactividad física y mental de los alumnos, por lo tanto, la tarea del docente "consiste en programar, organizar y secuenciar los contenidos de forma que el alumno pueda realizar un aprendizaje significativo, incluyendo los nuevos conocimientos en su estructura cognoscitiva previa y evitando, por tanto, el aprendizaje memorístico y repetitivo" Coll (1981, p. 86). Esta nueva perspectiva representa un requerimiento para el docente, que, por supuesto genera mucha inquietud en quienes se niegan a dejar su postura instruccionista para pasar a ser facilitadores y mediadores del proceso de aprendizaje, ya que además esto supone dejar de enfocarse en las propias formas de enseñar para centrar su atención en las formas en las que el niño aprende. Se requiere por tanto hacer una crítica a la propia práctica docente, desde el punto de vista pedagógico didáctico, ya que ahora la tarea no es la instrucción, sino la construcción del conocimiento a través de una práctica docente renovada y redefinida en términos de beneficios para el estudiante.

Ahora bien, siguiendo la idea central de este ensayo, es necesario destacar el rol del docente en el área de matemática, siendo que, debido a la naturaleza misma de esta ciencia pura, se requiere que la labor del docente se enfoque en el fomento de la reflexión, donde juega un papel fundamental la experiencia y la formación del docente para que reconozca la necesidad de transformar los métodos pedagógicos. Es decir, las creencias no cambian por decreto, por ello, cualquier programa orientado a cambiar la manera como se enseñan las 
matemáticas en el aula debe partir de entender las creencias de los profesores para luego propiciar un cambio (Lebrija, Flores y Trejos, 2010).

Las matemáticas parecieran tener dos concepciones diferentes, una para el currículo y otra para el docente, y ambas concepciones, forman parte de las creencias sobre cómo enseñar matemáticas y cómo las aprenden los alumnos. Las creencias que tienen principalmente efecto en las acciones que toman los maestros de matemáticas en su salón de clase pueden clasificarse en categorías que hablan de la relación del conocimiento matemático y del proceso de conocerlo (Muis, 2004).

Asimismo, se considera que los docentes del área de matemática centran su atención en estudiar los fundamentos de la validez de las teorías matemáticas, limitándose a observar y explicar los procesos matemáticos, dejando en segundo plano reconocer y finalmente los mecanismos de producir la enseñanza de tales procesos. Es así como al abordar la tarea de orientar el aprendizaje del saber matemático, se debe asumir que no se puede presentar descontextualizado, ya que de este modo no se genera la posibilidad de despertar en el estudiante el pensamiento crítico, de desandar el difícil camino que llevó a la formulación de las diferentes teorías y conceptos (Leiva Abdala y Marian Palliotto, 2011).

De esta forma se evidencia que el docente de matemática no debe tener características especiales, sin embargo, debe comprometerse a dinamizar los procesos, proveer de herramientas al estudiante para que logre el aprendizaje, esto mediante la motivación, convertirse más que en el profesor en un estímulo para el estudiante, mostrándole la otra cara de las matemáticas, las que pueden facilitarle la comprensión de todo cuanto lo rodea, incluso su utilidad para la vida.

Finalmente se requiere del docente que desarrolle nuevas habilidades, que sea capaz de organizar sus actividades de tal forma que se estimule el aprendizaje durante cada de los momentos y procesos del acto educativo, en este sentido es necesario que se actualice constante de manera que le proporcione mayor libertad al estudiante para que desarrolle su creatividad, es decir que le proporcionen un rol más activo al discente (Ministerio de Educación Nacional, 2015).

Por otro lado, es necesario que docente demuestre coherencia entre lo que dice y lo que hace, ya que los estudiantes se vuelven muy sensibles a este aspecto. Si un profesor tiene cierto discurso, sus actos deben ser el fiel reflejo de sus ideas. Caso contrario, los estudiantes perciben la incoherencia y se vuelven los críticos más duros de ella. Razón por la cual, es necesario que 
el docente tenga una continua práctica reflexiva sobre su propio discurso y sus acciones, siendo ejemplo, con su propia vida de que es posible siempre mejorar en credibilidad y así, convertirse en una persona en la cual, los estudiantes pueden confiar (Ortiz, 2015).

\section{CONCLUSIONES}

Cada uno de los planteamientos presentados anteriormente permiten afirmar lo que señala que los procesos educativos deben adecuarse a las formas de aprendizaje de los estudiantes, ya que no aprendes de la misma forma, debido a que no todos tienen la misma visión de todo aquello que los rodea. Es así como desde la didáctica de la matemática según el constructivismo los procesos de aprendizaje deben estar vinculados con las vivencias de los estudiantes, donde se tomen en cuenta sus conocimientos previos.

Por otra parte, las teorías estudiadas permiten afirmar lo que señala Vygotsky, en cuanto a la necesidad de buscar explicar la manera cómo influye el ambiente en el conocimiento de los individuos, en este caso de los estudiantes, de esta forma con base a sus postulados (Vygotsky), las personas con ayuda de instrumentos y signos dirigen su atención, organizan la memorización consiente y regulan su conducta. Lo que aunado a lo expuesto por Ausubel las experiencias de los estudiantes tienen influencia en sus aprendizajes si esto se sabe canalizar por parte del docente, en el caso de estudio aplicado al área de las matemáticas.

De lo que se deriva que la enseñanza de las matemáticas dada la relevancia que tiene para el desarrollo de los estudiantes debe estar dirigida a potenciar la zona de desarrollo próximo, es decir que sea una secuencia de los procesos donde se consideren los conocimientos previos, como fundamento para nuevo aprendizaje, donde se tenga en cuenta que los significados de las concepciones difieren unos de otros y dependen del contexto en el cual el estudiante se desenvuelva, ya que estos significados provienen del medio social, pero deben ser asimilados o interiorizados, en otras palabras se da de la interacción del sujeto con el objeto.

Ahora bien, el enfoque constructivista para la enseñanza - aprendizaje de las matemáticas se amerita un trabajo arduo que involucre a los actores del quehacer educativo sobre todo docentes y discentes, todo con el fin de transformar las ideas estratificadas y estandarizadas que se tienen sobre esta área. De ahí, que adoptar una postura constructivista no sólo permitirá advertir las dificultades que suelen tener los alumnos para aprender, sino que también aportará una guía para desarrollar un proceso de enseñanza donde el protagonista 
central es el alumno, considerando sus intereses, habilidades para aprender y necesidades en el sentido más amplio.

Por otra parte, es urgente cambiar o modificar las metodologías en el proceso educativo de las matemáticas replanteando los mecanismos para presentar los contenidos académicos, afianzándolos en las vivencias de los estudiantes, para que ellos puedan construir sus aprendizajes desde sus propias experiencias, entender la relación que existe entre lo aprendido en la escuela con lo que se vive en el hogar y en la comunidad, ya que considerando el postulado de Vygotsky el medio en el que se desenvuelve el estudiante es determinante para su desarrollo cognitivo.

En virtud de todo lo antes expuesto, se hace necesario reformular las formas cómo se han venido enseñando los contenidos del programa de matemática en las instituciones educativas del Municipio Zona Bananera del Departamento del Magdalena en Colombia, para que se aborden estrategias y metodologías que contemplen por un lado, una visión holística del proceso educativo, es decir que considere los aspectos, sociales, culturales, económicos y políticos, donde se desarrolla el acto educativo y por otro lado, los aspectos humanos del estudiante, pero sobre todo donde el estudiante viva su aprendizaje, lo integre a su vida como una herramienta funcional para descubrir, conocer y procesar la información de todo cuanto lo rodea.

Además de contar con docentes que actúen como orientadores o facilitadores en el proceso de enseñanza-aprendizaje, con metodologías principalmente de construcción, donde el papel principal lo tenga el estudiante como un ente activo que debe pensar, deducir, contrastar y buscar las soluciones adecuadas dependiendo de la situación de aprendizaje a la cual sea sometido. Un docente que entregue la libertad de crear, construir a sus alumnos para que éstos edifiquen su propio aprendizaje utilizando todas sus habilidades, destrezas, necesidades e intereses como seres dotados de razonamiento lógico matemático.

\section{REFERENCIAS}

Abbott, S. (1999). Teorías de aprendizaje. Revista encuentro educacional Luz.

Abdala, L., y Palliotto, M. (2011). Un enfoque constructivista en la enseñanza y el aprendizaje de la matemática para el desarrollo de competencias.Revista RDEHECS, 11(6), 91-113. https://dialnet.unirioja.es/descarga/articulo/4172063.pdf 
Albornos, L. (2017). Competencias tecnológicas para potenciar las habilidades lógicas matemática en los estudiantes de educación media general del Municipio Mara. Tesis de maestría, Universidad del Zulia, Venezuela.

Ausubel, D.P., Novak, J.D. y Hanesian, H. (1983). Psicología educativa. Un punto de vista cognoscitivo. México: Trillas

Blanco, K. (2014). El constructivismo como fundamento pedagógico para la enseñanza de las matemáticas. (Tesis de maestría, Universidad del Zulia).

Carrillo, J. (2000). La formación del profesorado para el aprendizaje de las matemáticas. Revista Uno. http://ocenet.oceano.com/consulta/welcome.doc

Coloma, C., y Tafur, R. (1999). El constructivismo y sus implicancias en educación. Educación, $8(16)$ 217-244.

http://revistas.pucp.edu.pe/index.php/educacion/article/view/5245/5239

Coll, C. (1997). El constructivismo en el aula. Barcelona. Editorial Grao

Clemente, L. (2010). La construcción del conocimiento matemático: modelo pedagógico constructivista en las instituciones educativas de Santa Marta, Colombia. (Tesis doctoral, Universidad Rafael Belloso Chacín). Maracaibo - Venezuela

González, J. (2017). La enseñanza de las matemáticas bajo perspectiva de modelos innovadores. (Tesis de maestría) Universidad Rafael Belloso Chacín. (URBE).

Lebrija, A., Flores, R. y Trejos, M. (2010). El papel del maestro, el papel del alumno: un estudio sobre las creencias e implicaciones en la docencia de los profesores de matemáticas en Panamá. Educ. http://www.scielo.org.mx/scielo.php?script=sci_arttext\&pid=S1665-58262010000100003

Medina Castañeda, Y. (2011). El constructivismo y la realidad matemática. Universidad Corporación Unificada Nacional de Educación Superior.http://www.etnomatematica.org/publica/articulos/ARTICULO\%20EL\%20CONSTRUCTIVISMO\%20Y\%20LA\%20REALIDAD\%20\%20MATEMATICA2015-YAMILE-\%20-\%20copia.pdf

Méndez, A. (2002). Constructivismo social. Artículo curso 1 de Epistemología, Centros Comunitarios de Aprendizaje de Universidad Virtual del Tecnológico de Monterrey, México. http://www.cca.org.mx/dds/cursos/cep21/modulo_1/main0_35.htm

Ministerio de Educación Nacional (2015b), Bases para la Construcción de los Lineamientos de 
Política Pública del Sistema Nacional de Educación Terciara (SNET), Viceministerio de Educación Superior, Dirección de Fomento de la Educación Superior, Ministerio de Educación Nacional, Bogotá.

Muis, K. (2004), "Epistemología personal y matemáticas", Review of Educational Research, 74(3).

Ortiz (2015). El constructivismo como teoría y método de enseñanza. Sophia, Colección de $\begin{array}{lllll}\text { Filosofía de } & \text { la } & \text { Educación, } & \text { 93-110. }\end{array}$ http://www.redalyc.org/pdf/4418/441846096005.pdf

Payares, J. (2016). Modelo constructivista para el desarrollo de competencias científicas mediante el uso de las tecnologías en las instituciones educativas de municipio Tenerife del departamento del Magdalena. Tesis doctoral, Universidad Rafael Belloso Chacín, Venezuela.

Piaget, J. (1978). La equilibración de las estructuras cognitivas. Madrid: Siglo XXI.

Pozo Jaramillo, P. (2013). Propuesta metodológica para el aprendizaje de la contabilidad, desde una perspectiva constructivista dirigida a los docentes de la escuela de ciencias administrativas y contables de la Pontificia Universidad Católica del Ecuador Sede Santo Domingo. http://repositorio.puce.edu.ec/bitstream/handle/22000/5688/T-PUCE5837.pdf? sequence $=1 \&$ is Allowed=y .

Salomón Contreras, G. (2010). Rol del docente desde la perspectiva constructivista. Tesis de maestría, Universidad Rafael Belloso Chacín, Venezuela.

Suárez, M. (2012). El constructivismo y los procesos educativos en las instituciones educativas del municipio Maracaibo. Tesis de maestría, Universidad Rafael Belloso Chacín, Venezuela.

Vargas, E. (2006). Constructivismo y modernización del aprendizaje. México: Enciclopedia de la Psicopedagogía.

Vygotsky, L. (1978). El desarrollo de los procesos psicológicos superiores. Primera edición. Editorial Crítica del grupo Editorial Grijalbo. Barcelona. 